\section{QUANPLUS: A Spreadsheet for Calculation of Heritability and Generation Mean Separation}

\author{
Richard H. Ozminkowski, Jr., Robert H. Moll' ${ }^{1}$, and \\ Randolph G. Gardner ${ }^{3}$ \\ Department of Horticultural Science, Box 7609, North Carolina State \\ University, Raleigh, NC 27695-7609
}

Additional index words. quantitative analysis, breeding, microcomputers

Quantitative traits are often time-consuming to analyze. A spreadsheet has been designed for computers running MS-DOS to quickly estimate heritabilities and additive genetic variance of a quantitative trait for an inheritance study (Ozminkowski et al., 1990). It is also useful for educational purposes. It is based on a six-generation analysis that includes two inbred lines as parents, one with the trait in question (P1) and the other without (P2), the $\mathrm{F}_{1}$ hybrid (F1), a backcross of the $\mathrm{F}_{1}$ to each parent $(\mathrm{BC} 1, \mathrm{BC} 2)$, and the $\mathrm{F}_{2}$ population (F2).

The spreadsheet requires Lotus 123, ver. 2 (Cambridge, Mass.), or a compatible spreadsheet program. QUANPLUS can analyze discrete or continuous data. Raw data are entered for discrete variables (e.g., subjective ratings, percentages). Figure 1 shows an analysis of discrete data collected on a 1 to 5 rating scale. In analysis of discrete variables, values entered into the data matrix must be the quantity of plants given each rating value [i.e., 11 P1 plants given a 4 rating (Fig. 1)]. Sample variances of discrete data are calculated in hidden columns without the@var subroutine of 123. The data matrix for discrete analysis can be adjusted to accommodate other rating scales or whole number percentages. Analysis of a continuous variable (e.g., yield) requires the number, means, and variances of samples,

The arithmetic mean of the variances of the inbred parents and $F_{1}$ samples serves to estimate the environmental variance (@SQR E). The $F_{2}$ variance represents the phenotypic variance (sVar F2). The genotype-environment interaction is assumed to be zero. This assumption allows estimation of the ge-

Received for publication 7 Sept. 1990. The research reported in this publication was funded, in part, by the North Carolina Agricultural Research Service (NCARS), Raleigh. The use of trade names in this publication does not imply endorsement by NCARS of the products mentioned nor criticism of those omitted. The cost of publishing this paper was defrayed in part by the payment of page charges. Under postal regulations, this paper therefore must be hereby marked advertisement solely to indicate this fact.

'Graduate Research Assistant. Present address: Dept. of Horticulture, The Ohio State Univ., Columbus, $\mathrm{OH} 43210$

'Professor of Genetics.

Associate Professor. dom are calculated, but significance must be determined manually using a Student's t table (Steel and Torrie, 1980)

Another scaling spreadsheet, JNTSCALE ( $\mathrm{Ng}, 1990)$, is available, but means are not separated and population (not sample) variances must be entered; population variances are also produced by QUANPLUS for use in JNTSCALE. QUANPLUS.WK1 is available for R.H.O. or R.G.G. for a nominal charge or on HortLine, the ASHS BBS (703/8362418). The spreadsheet is also available for use with Microsoft Excel (Redmond, Wash.) on a Macintosh (Apple Corp., Cupertino, Calif.) microcomputer (from R.H.O).

\section{Literature Cited}

netic variance (@SQR G = sVar F2 @SQR E) (Wright, 1968). The additive component of the genotypic variance (@SQR A) is estimated using the variances of the $F_{2}$ and two backcross generations (Warner, 1952). Narrow- and broad-sense heritabilities are calculated as described by Warner (1952) and Fehr (1987), respectively. If backcross generations are unavailable, all parameter estimates, except those of @SQR A and narrow-sense heritability, remain valid.

Means of each generation are compared using a Student's $t$ test with unequal variances (Steel and Torrie, 1980). The $F_{1}$ vs. mid-parent, $F_{2} v s$. mid-parent, and $F_{2} v s$. the mean of both backcrosses are also tested as verification of complete additivity of the trait (Wright, 1968). Effective degrees of free-
Fehr, W.R. 1987. Principles of cultivar development. vol. 1. Macmillan, New York. p. 95105 , crocomputer. HortScience 25:363.

Ozminkowski, R.H., Jr., R.G. Gardner, R.H. Moll, and W.R. Henderson. 1990. Inheritance of prostrate growth habit in tomato. J. Amer. Soc. Hort. Sci. 115:674-677.

Steel, R.G.D. and J.H. Torrie. 1980. Principles and procedures of statistics. 2nd ed. McGrawHill, New York. p. 106, 577.

Warner, J.N. 1952. A method for estimating heritability. Agron. J. 44:427-430.

Wright, S. 1968. Evolution and the genetics of populations. Vol. 1: Genetic and biometric foundations. University of Chicago Press, Chi-
Ng, T.J. 1990. Generation means analysis by micago. p. 391-403.

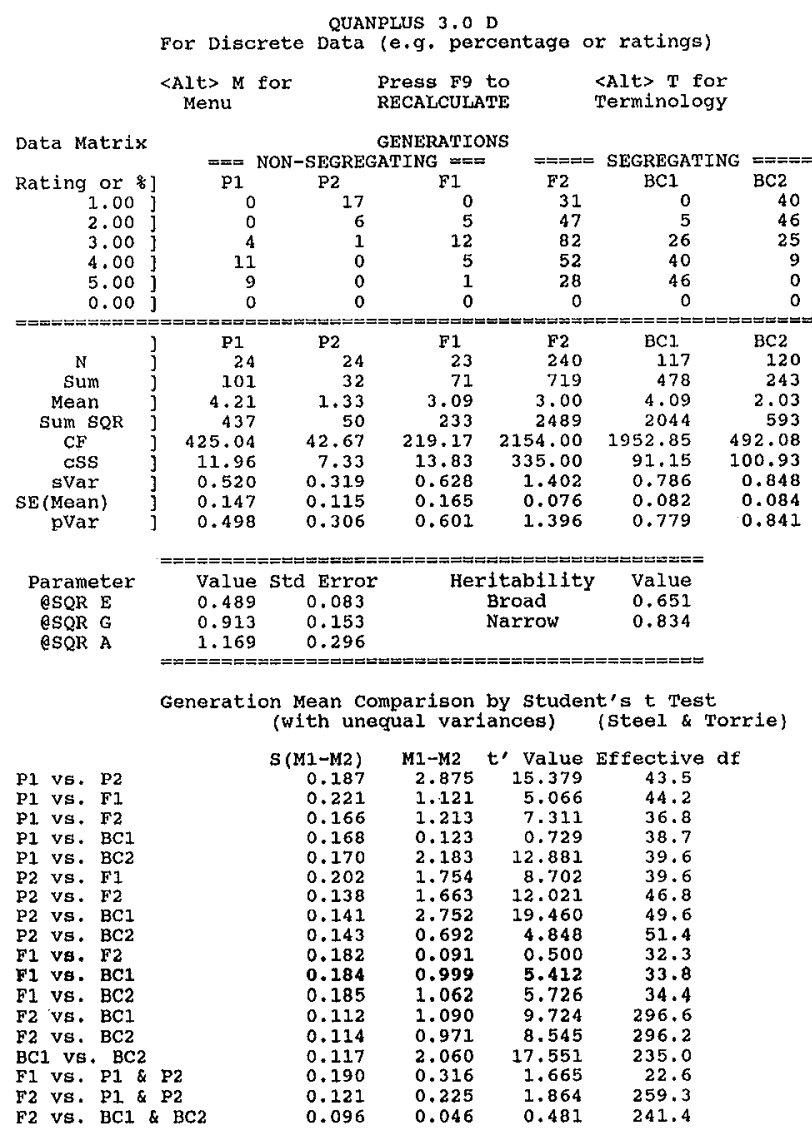

Fig. 1. Sample output from QUANPLUS.WK1 with a discrete variable. 